\title{
1 Antibiotics shift the temperature response curve of Escherichia coli growth
}

3 Mauricio Cruz-Loya ${ }^{1 *}$, Elif Tekin ${ }^{1,2^{*}}$, Tina Manzhu Kang ${ }^{2 *}$, Alejandra Rodriguez-Verdugo ${ }^{3}$, Van

4 M. Savage ${ }^{1,2,4}$, and Pamela J. Yeh ${ }^{2,4 * *}$

5

* Authors contributed equally. Order of co-first authors was decided by mutual agreement.

$8 \quad$ Corresponding author pamelayeh@ucla.edu

$11{ }^{1}$ Department of Computational Medicine, David Geffen School of Medicine, University of

12 California, Los Angeles, CA 90095, U.S.A.

13

$14{ }^{2}$ Department of Ecology and Evolutionary Biology, University of California, Los Angeles, CA

1590095 , U.S.A.

16

$17{ }^{3}$ Department of Ecology and Evolutionary Biology, University of California, Irvine, CA 92697,

$18 \quad$ U.S.A

19

$20{ }^{4}$ Santa Fe Institute, Santa Fe, NM 87501, U.S.A. 


\section{Abstract}

34 Temperature variation — through time and across climatic gradients — affects individuals,

35 populations, and communities. Yet how the thermal response of biological systems is altered by

36 environmental stressors is poorly understood. Here we quantify two key features - optimal

37 temperature and temperature breadth - to investigate how temperature responses vary in the

38 presence of antibiotics. We use high-throughput screening to measure growth of Escherichia coli

39 under single and pairwise combinations of 12 antibiotics across seven temperatures that range

40 from $22^{\circ} \mathrm{C}$ to $46^{\circ} \mathrm{C}$. We find that antibiotic stress often results in considerable changes in the

41 optimal temperature for growth and a narrower temperature breadth. The direction of the optimal

42 temperature shifts can be explained by the similarities between antibiotic-induced and

43 temperature-induced damage to the physiology of the bacterium. We also find that the effects of

44 pairs of stressors in the temperature response can often be explained by just one antibiotic out of

45 the pair. Our study has implications for a general understanding of how ecological systems adapt

46 and evolve to environmental changes.

48 Key words: temperature response, thermal variation, antibiotics, multiple stressors, climate

49 change, Escherichia coli 


\section{Introduction}

52 Many environments experience daily and seasonal temperature fluctuations that affect rates of

53 physiological processes. These changes in turn affect biological and ecological traits and

54 ultimately impact the behavior of communities (1-9). In this manner temperature fluctuations can

55 drive the evolution of organisms through variation in thermal sensitivity - the ability to function

56 and survive at different temperatures $(2,10-15)$.

Measuring the growth of a living organism at different temperatures yields a temperature response curve (Figure 1a). Typically, temperature response curves have a single peak, corresponding to an optimal temperature where growth is maximized (2). As the temperature changes away from the optimum in either direction, the growth rate decreases, with an especially steep decline at higher temperatures. The range of temperatures in which an organism can grow to a certain extent (e.g. at least half of the maximum growth) is termed the temperature breadth. Living organisms are said to experience either cold or heat stress at extreme temperatures where growth is substantially less than optimum.

Thermal response curves are fundamental to grasping the variability of physiological and ecological traits in response to temperature changes in different taxonomic groups and habitats. Because shifts in the thermal response curves are representative of average fitness performance and temporal niches (16), optimal temperature and thermal breadth are indicative of evolution and acclimation patterns based on how species' performance contributes to survivorship or

72 fecundity (14). For instance, seasonal variation in temperature could lead to an evolution of

73 different attack and escape speeds that would allow individuals to perform best when they are

74 predator or prey (17).

At a cellular level the performance of an organism across different temperatures can lead to

77 various genetic and physiological adaptation mechanisms. For example, in bacteria thermal

78 sensitivity is related to many physiological and genetic modulations in metabolism, including

79 outer membrane rigidity $(18,19)$, chemotaxis $(20,21)$, enzymatic thermo-stability $(22,23)$, and 
other general adaptive responses $(24,25)$. Moreover, the heat shock response - a cellular mechanism to deal with the deleterious effects of high temperatures, such as protein misfolding and aggregation - is highly conserved in both prokaryotes and eukaryotes $(25,26)$.

83 Understanding responses to temperature changes is important to infer general patterns of how

84 organisms, species, communities, and ecosystems are adapting to fluctuations in climate patterns and different environmental conditions.

Any environmental feature that kills a living organism or reduces its growth can be considered a stressor. Temperature can interact with other environmental stressors such as light, precipitation, $\mathrm{pH}$, and salinity. Exposure to different stressor types and intensities can lead to phenotypic variation in an organism's ability to respond to temperature changes $(27,28)$. Nevertheless, how

91 the effects of environmental stressors interact with temperature responses is not well understood.

92 Therefore, insights on whether temperature responses - as described by optimal temperatures

93 and temperature breadths - can change rapidly and plastically in the presence of other

94 environmental stressors have been lacking. In fact, it has been commonly assumed that thermal

95 responses are not altered in the presence of other stressors (29-31).

97 A systematic approach that informs how optimal temperatures and temperature breadths are

98 shifted by stressors (Figure 1) is needed to uncover these ambiguities and provide additional

99 insights on fitness tradeoffs and thermal adaptation strategies. Here, we use a combined

100 empirical-theoretical approach to study if the characteristics of thermal response curves change

101 in response to additional environmental stressors. In particular, we use an experimental system of

102 Escherichia coli and antibiotics as stressors in order to investigate how a physiological trait-

103 growth of the bacterium - responds to variation in temperature in the presence of different

104 stressor conditions. We obtain temperature response data for E. coli in 12 single-drug and 66

105 two-drug combination environments, where antibiotics are chosen to cover a wide range of

106 mechanisms of action (Table 1). We then quantify both the optimal temperature and the

107 temperature breadth of $E$. coli in the presence of these different environments. 
We first show that individual stressors can have a substantial impact on the optimal temperature

110 and temperature breadth. Next, we evaluate if the directions of the shifted thermal responses are

111 related to the mechanism of action of the antibiotics. Previously, we determined that some

112 specific classes of antibiotics have similar physiological effects to either heat or cold stress in $E$.

113 coli (32). This classification was based on comparing the experimentally determined interaction

114 profile (synergies and antagonisms with other stressors) of antibiotics and various growth

115 temperatures (Table 1). We find that in most cases the direction of the shifts in the thermal

116 responses under antibiotic stress can be explained through these groups. Lastly, we investigate

117 how pairs of stressors move optimal temperatures in different directions as compared to the

118 optimal temperatures under single-stressor conditions. In particular, we evaluate the extent to

119 which the optimal temperatures result from integrated effects of both stressors, or whether a

120 single stressor is the key driver of the temperature response. We infer from our results that a

121 single drug often plays a dominant role in determining the optimal temperature response of a

122 combined treatment.

124 Our experimental and theoretical framework on temperature response curves of E. coli presented

125 here allows us to better understand how thermal sensitivities change in response to stressors.

126 Therefore, our analysis will shed light on fundamental features shaping the ecological and

127 evolutionary responses of organisms facing complex environmental conditions. By using

128 antibiotics as stressors and a bacterium as a model organism, our study system is particularly

129 valuable for its experimental tractability and reproducibility.

\section{Results}

132 In this paper, we investigate how different stressors (antibiotics) alter an organism's response to

133 temperature, both in isolation and in combination. To do this, we determine the temperature

134 optimum and temperature niche/breadth of $E$. coli by fitting the extended Briere model

135 (developed here, see Materials and Methods) to experimental data of bacterial growth collected

136 under different (unstressed and stressed) growth environments across multiple temperatures

$137\left(22^{\circ} \mathrm{C}, 25^{\circ} \mathrm{C}, 30^{\circ} \mathrm{C}, 37^{\circ} \mathrm{C}, 41^{\circ} \mathrm{C}, 44^{\circ} \mathrm{C}, 46^{\circ} \mathrm{C}\right)$. The entire dataset and model fits are shown in 
138 Supplemental Figure 1. More details about the model and fitting procedure can be found in the

139 Materials and Methods and Supplemental Information.

141 First, we explore how the optimal growth temperature of E. coli changes under single-stressor

142 conditions (Figures 1a, 1b). We find that the majority of the single-drug environments exhibit

143 left shifts - meaning the optimal temperature is lower-(Figure 1c) compared to the no-drug

144 condition, $T_{o p t}=37.7^{\circ} \mathrm{C}, \mathrm{CI}:\left(36.7^{\circ} \mathrm{C}, 38.6^{\circ} \mathrm{C}\right)$. Right shifts are both less common and of lower

145 magnitude than the observed left shifts. In addition, we find that the thermal niche breadth

146 typically becomes narrower under antibiotic stress, meaning that E. coli can survive and properly

147 function at a reduced temperature range.

149 Next, we investigate whether the physiological effects of antibiotics bear any relation to the

150 direction of the observed shifts in the temperature responses (Figure 2). To do this, we group the

151 antibiotics according to the similarity of their physiological effects to those of low or high

152 temperatures, as determined previously (32). We observe the direction of the shifts for both

153 single drugs (Figure 2a, left panels) and drug combinations that contain one or more of the

154 antibiotics in the group (Figure 2a, right panel). We find that - for both single drugs and

155 combinations - cold-similar antibiotics (i.e., with effects on bacteria similar to those caused by

156 low temperatures) tend to either leave the optimal temperature unchanged or shift it slightly to

157 the right (i.e., to higher optimal temperatures). In contrast, heat-similar antibiotics (i.e., with

158 effects on bacteria similar to those caused by high temperatures) tend to result in unchanged

159 optimal temperatures or shifts to the left (i.e., to lower optimal temperatures). In fact, bacteria

160 exposed to aminoglycosides (TOB, GEN, STR), which induce misfolding of membrane proteins

161 and have similar physiological effects to very high temperatures (Figure 2), show the greatest

162 shifts towards the left. This is not the case for other protein synthesis inhibitors such as ERY or

163 CLI that are similar to cold. Interestingly, beta-lactams shift the temperature curves in a similar

164 way to heat-similar drugs when used in combinations, despite them having a different

165 mechanism of action (inhibition of cell wall synthesis) that was not found to be heat-similar. 
167 We then compare the optimal temperature and temperature niche - the range between the

168 temperatures that result in half-maximum growth — for bacteria under all antibiotic combinations

169 to the single drug conditions (Figure 2b). For some antibiotics (e.g., ERY, CPR) the optimal

170 temperature and the thermal niche range are similar to those of the single drug when combined

171 with most other antibiotics. In contrast, there are other antibiotics for which these features show

172 much more variation when combined with others (e.g. GEN, STR, TOB, FOX). This suggests

173 that some antibiotics may act as the main drivers of the temperature response curve of antibiotic

174 combinations.

175

176 Following this idea, we further explored how the optimal growth temperature is determined

177 under combinations of stressors relative to the optimal temperature under single stressor

178 conditions. We contrast the observed optimal temperatures with the predictions of five candidate

179 models of how the combination optimal temperature could be determined from that of the single

180 stressors (see Material and Methods, Figure 3a). The min and max models assume that the

181 optimal temperature of the combination is determined by the optimal temperature of a single

182 drug (the minimum or the maximum of the pair, respectively). These models best describe most

$183(65 \%)$ multi-drug combinations (Figure 3b). The attenuated and elevated models assume that

184 the optimal temperature of the combination is either lower or higher, respectively, than for both

185 single drugs. These models best describe $18 \%$ of the combinations. Lastly, the mean model

186 assumes that the temperature of the combination is determined by the average of the single drug

187 optimal temperatures. This model best described only $17 \%$ of the drug combinations. These

188 results suggest that the optimal temperature of antibiotic combinations is often determined by a

189 single drug.

190

191 Finally, we explore cases where single-driver models (min, max, attenuated, and elevated)

192 represent the best optimal temperature model over the mean model, where both stressors

193 compromise to result in the optimal temperature of an organism in the presence of combined

194 stressors (Figure 3c). Interestingly, we rarely observe aminoglycosides (GEN, STR, TOB),

195 antibiotics similar to high-heat, being drivers. In contrast, some cold-similar drugs (ERY, LVX,

196 CPR), but not all (CLI, TET), frequently drive the optimal temperature of the combination. To 
197 account for the possibility that some antibiotics appear to be a driver more often than others

198 purely by chance, we used a permutation test to evaluate our data against the null model that all

199 drugs are equally likely to be a driver (see D-statistic in Supplemental Information for details).

200 This test provides strong evidence $(\mathrm{p}=0.002)$ that some antibiotics have a greater tendency to be

201 drivers than others by testing the entire dataset simultaneously. We also tested if specific

202 antibiotics are drivers more often than expected by chance (see M-statistic in SI). However, we

203 did not obtain statistically significant results for individual drugs, after correcting for multiple

204 comparisons. We believe this may be due to a lack of statistical power to detect differences due

205 to the smaller number of observations for individual antibiotics when compared to the full

206 dataset.

208 Discussion

209 Through a systematic analysis of growth response curves of bacteria across different

210 temperatures and under different stressor environments, we investigate the effects of stressors on

211 the phenotypic variation in temperature response traits - optimal temperature and temperature

212 breadth. We see that stressors often decrease the temperature breadth and shift the optimal

213 temperature in a direction that depends on their physiological mechanism of harm. In addition,

214 our results suggest that left shifts - where the optimal temperature in a stressed environment is

215 lower than the optimal temperature in unstressed environmental conditions - are more common

216 and dramatic as opposed to right shifts towards higher optimal temperatures. This may be

217 partially due to the asymmetry of temperature response curves, where the interval between the

218 minimum and optimal growth temperatures is larger than that between the optimal and maximum

219 growth temperatures.

221 High temperature harms living organisms through multiple mechanisms, including misfolding

222 and aggregating proteins, damaging nucleic acids, and increasing membrane permeability (25).

223 The heat-shock response attempts to prevent and/or repair this damage by producing chaperones

224 that aid the correct folding of proteins (33). It has previously been shown that certain kinds of

225 antibiotics can activate components of the heat-shock response $(32,34)$. However, adding

226 antibiotics to heat stress is unlikely to help the cell survive high temperatures, since the heat- 
shock response is already induced by the high temperature alone. Thus, right shifts in the optimal

228 temperature may be rare because it is unlikely that adding a second stressor can reduce or repair

229 the high-temperature induced damage. In most cases where we do observe a right shift, it seems

230 to be due to asymmetrical effects on the temperature response curve, where the left portion (i.e.

231 below the unstressed optimal temperature $T_{o p t}$ ) is more depressed by the antibiotic than the right

232 portion (Figure 2a, Supplemental Figure 1).

234 In contrast, cold temperatures predominantly slow down cell growth by suppressing DNA

235 replication or protein translation $(35,36)$. Since the effects of low temperature seem to be

236 primarily mediated by slowing down metabolism and growth rather than the accumulation of

237 physical damage, it seems more likely that stressors can shift the optimal temperature to the left,

238 especially when the stressor is more harmful at higher temperatures. In some cases, cold

239 temperatures might allow cells to sustain antibiotic killing because certain antibiotics are only

240 effective against actively growing cells (37). Low temperatures have also been shown to alter the

241 structural stability (38) or the global uptake of some antibiotics such as gentamicin, thus

242 impairing killing efficiency (39).

244 Based on network clustering methods $(40,41)$, we previously found that certain antibiotic classes 245 have similar physiological effects to either heat or cold in E. coli (32). These temperature-drug 246 groups were also shown to correlate with changes in drug sensitivity of high-temperature adapted

247 strains obtained by Rodríguez-Verdugo et al (42). Interestingly, here we find that in most cases

248 the direction of the shifts in the optimal temperature can be predicted from these groups. Cold-

249 similar drugs tend to either leave the optimal temperature unchanged, or to shift it slightly to the

250 right. In contrast, heat-similar drugs tend to result in larger shifts to the left or leave the optimal

251 temperature unchanged (Figure 2). Similar trends are exhibited by antibiotic combinations

252 containing drugs in these groups.

254 We propose a shared-damage hypothesis to explain this phenomenon: antibiotics that damage the 255 same cellular functions as temperature stress (heat or cold) will cause an increased burden to the 256 cell machinery that repairs this damage. For example, simultaneous exposure to aminoglycosides 257 and high temperatures will result in more misfolded proteins than either stressor on its own. 
Upon addition of an antibiotic, the stress-response machinery of the cell could be overwhelmed at less extreme temperatures, causing a greater reduction in growth at temperatures that cause similar physiological damage to the drug. The effect of these kinds of antibiotics in the temperature response curve will thus be asymmetrical. Growth will be more strongly reduced in

262 the direction (heat or cold relative to Topt) where the drug and temperature damage overlap, and

263 the optimal temperature will often shift towards the opposite direction because it suffers less

264 from growth reduction. The cases with the most pronounced shifts in optimal temperature tend to

265 have lower peak growth (Figure 2a). This suggests that perhaps these shifts become more

266 pronounced when increasing the antibiotic concentration. Hence, we hypothesize that increasing

267 the concentration of heat-similar drugs will result in greater shifts to the left and that doing so for 268 cold-similar drugs will result in greater shifts to the right.

Notably, although the aminoglycosides (TOB, GEN, STR) share the same cellular target - the ribosome - as the other protein synthesis inhibitors (CLI, ERY, TET) used in our study, they

272 result in distinct effects on the thermal response. Previously, differences in the effects of

273 aminoglycosides and other protein synthesis inhibitors at different growth rates have been

274 attributed to the reversibility of ribosomal binding (43). In that study, the authors found that STR

275 is more effective when the growth rate is lower, which does not agree with our results at low

276 temperatures. This discrepancy may be because the reduction in growth was previously

277 manipulated by nutrient limitation as opposed to the temperature variation in our study. mechanisms of action being qualitatively different, with the aminoglycosides being heat-similar

281 and the other protein synthesis inhibitors being cold-similar. This is because aminoglycosides,

282 unlike other protein synthesis inhibitors, induce mistranslation by the ribosome that decreases

283 translational accuracy and causes protein misfolding (44). Cold temperatures may counteract this

284 effect by slowing down ribosomal activity and increasing accuracy (45), thus causing

285 aminoglycosides to be less effective when bacterial growth is suppressed at lower temperatures,

286 as we observe. Reduced drug uptake at low temperatures could also play a role (39). 
Interestingly, beta-lactams have a similar effect in the temperature response as heat-similar drugs. We speculate that this may be due to increased effectiveness at high temperatures due to a synergy between the cell-wall damage caused by the antibiotic and the increased membrane permeability caused by high temperatures. Further disentangling these processes in future studies will help increase our understanding of the connection between antibiotic susceptibility and bacterial physiology.

The breadth of the temperature niche is typically reduced in the presence of antibiotics, both in isolation and in combination. The lower and upper limits of growth are believed to be set by chemical and physical limits on the biological processes necessary for bacterial physiology, growth, and cell division (2). Since drugs introduce additional physiological damage in addition to that caused by temperature extremes, it seems likely that in most cases antibiotics would further constrain the temperature response and kill off barely-surviving populations at the extreme temperatures. Previous studies have also observed the temperature niche being reduced upon exposure to stressors $(46,47)$. From our results, it is apparent that stressors can reduce the temperature niche of living organisms at either temperature extreme. Thus, species that experience a wide range of stressful conditions at different times could perhaps experience selection for a broader temperature breadth that can be adapted to various environmental stressors (16).

The temperature niche is measured as the range between the low and high temperatures for halfmaximum growth: its definition is therefore relative to the maximum growth. Consequently, conditions that decrease the right and central (i.e., near Topt) portions of the curve more than the

311 left portion can result in a temperature niche that is shifted to the left (and vice versa) in the

312 absence of increased growth at temperature extremes. These effects can lead to apparently

313 surprising cases where adding a drug extends the limits for the thermal niche of the unstressed

314 condition without increased growth at temperature extremes. This suggests that microbial

315 communities may experience shifted thermal niches - giving rise to a different competitive

316 landscape (e.g., due to reduced invasibility of high temperature habitats under

317 aminoglycosides) — in the presence of certain antibiotics. These effects could be particularly

318 important in the presence of variation in adaptations to antibiotics within microbial communities, 
which might cause the severity of their effects on the temperature curves to be species dependent.

322 We find that in most cases the shift in optimal temperature of $E$. coli due to antibiotic pairs is

323 primarily determined by a single antibiotic. However, we also found cases where interactions

324 between antibiotics seem to be important for determining the optimal temperature. For example,

325 aminoglycosides (TOB, GEN, STR) show the largest degree of downshifting of the optimal

326 temperature. However, when a second drug is added, this downshifting tends to be alleviated.

327 Thus, in combinations of stressors, aminoglycosides are not the dominant driver for changing

328 optimal temperature despite their large effects when used alone. When a shift in the thermal

329 optimum is alleviated by addition of a second antibiotic, this does not imply that the reduced

330 growth is also alleviated. Typically, these shifts are due to the second antibiotic decreasing some

331 regions of the temperature response curve more sharply than others (Supplemental Figure 1). A

332 notable exception involves interactions between certain aminoglycosides (GEN, STR) and other 333 protein synthesis inhibitors (ERY, TET and other aminoglycosides), possibly because inhibition

334 of protein synthesis reduces the aminoglycoside induced production of misfolded protein

335 aggregates.

337 The growth response to multiple environmental factors such as temperature, $\mathrm{CO}_{2}$, and $\mathrm{pH}$ has 338 been measured in green algae (48). In conditions with a large number of factors present simultaneously, the response is dominated by a single, severely detrimental driver. In contrast, in environments with a smaller number of factors, specific interactions between drivers were found

341 to determine overall growth rather than the response to an overriding factor. These results were

342 explained by the authors by the presence of a severely detrimental driver limiting the growth

343 reduction that can be obtained by additional stressors, making the severe driver the primary

344 determinant of the response. In contrast, we find that the effects of an antibiotic can sometimes

345 be partially undone by another (e.g. aminoglycosides and other protein synthesis inhibitors). This

346 suggests that, while identifying a dominant environmental driver can be a simplified approach to

347 understanding organismal response to a complex system, this needs to be done with care since

348 interactions between drivers can be a contributing factor as well. 
351 The thermal optimum is often below the mean environmental temperature. This is because

352 thermal response curves typically decrease sharply at high temperatures, so the penalty of going

353 above the optimal temperature is much steeper than going below. Consequently, the exact

354 distance between the thermal optimum and the mean is determined by the temperature variability

355 in the environment (29-31). It is thus often tacitly assumed that the optimum temperature of

356 individuals closely aligns the environment in which the individual has been reared and/or the

357 species has evolved.

359 For this reason, the optimal temperature is not expected to quickly shift in response to other 360 stressful conditions. This is a common assumption in mathematical models that describe the 361 combined effects of temperature with other stressors such as $\mathrm{pH}$ (49), nutrient limitation (50),

362 and humidity (51). In contrast, we observe that stressors can substantially and quickly change the 363 optimal temperature for growth of a bacteria. A study that evaluated the combined effects of 364 temperature and salt in slime molds (52) also found shifts in the thermal optimum, suggesting 365 that this phenomenon is not limited solely to antibiotics.

367 Any physical or chemical environmental feature that kills or slows the population growth of a 368 living organism can be considered a stressor. Antibiotics are stressors to bacteria in clinical 369 settings but they may not always take this role in nature. It has been proposed that some 370 antibiotics may participate in communication or be byproducts of metabolism in their natural 371 environments (53-55). Since we explain thermal optimum shifts through differential growth 372 reduction, our shared-damage hypothesis predicts antibiotic-induced thermal optimum shifts will 373 occur when antibiotics are acting as stressors. However, this will not necessarily happen when 374 antibiotics have a different role (e.g. communication) at much lower concentrations than those 375 relevant in the clinic. In these cases, we would expect thermal optima to change only if there is a 376 non-negligible fitness decrease caused by the antibiotic. Further work could test this by 377 measuring the effects of an antibiotic on the thermal responses of microbes that naturally occur 378 in the same environments as the antibiotic. 
An exciting potential application of the shared-damage hypothesis is in predicting the effect of other stressors on the thermal optima of living organisms. To do this, further studies are necessary to evaluate the extent to which the physiological damage caused by other environmental stressors - such as pressure and $\mathrm{pH}$ - is similar to either temperature stress or

384 antibiotics. This can be done by comparing either the gene-expression profile or the interaction profile (i.e. synergies and antagonisms with other stressors) of the environmental stressor of interest with those of extreme temperatures and/or antibiotics, as has been done to explore the overlap between antibiotics and temperature $(32,34)$. Our hypothesis would then predict that stressors that induce similar damage to high temperature will result in left shifts in $T_{\text {opt }}$ (and vice versa). Moreover, the direction of the shift induced by a stressor should be the same as that of

390 other stressors (e.g. antibiotics) that cause similar physiological damage. For example, beta-

391 lactam antibiotics compromise the integrity of the bacterial cell wall so we speculate that the 392 induced damage to the cell could have certain similarities to osmotic shock. If this were true, it 393 seems possible that osmotic shock might change the temperature responses in a similar way to 394 beta-lactams.

Although there has been substantial interest in understanding thermal response curves because of their potential to predict responses to climate change $(17,56,57)$, the implications might be even

398 broader. For example, an intriguing recent study showed that increased local temperatures were 399 associated with increasing antibiotic resistance (58). This may be because temperature or 400 seasonality effect environmental growth of resistant strains $(59,60)$ and horizontal gene 401 transfer - one method of facilitating resistance transmission $(61,62)$. Another study showed that 402 adaptations to long-term temperature changes unexpectedly coincided with mutations conferring 403 resistance to rifampicin, an antibiotic that impairs RNA polymerase (42). Climate change has 404 also been linked to changes on host-parasite dynamics that alter the frequency and severity of 405 many infectious diseases $(63,64)$. Our work here and elsewhere shows that certain classes of 406 antibiotics are more effective at different temperatures, and that there is substantial overlap in the 407 response mechanisms to temperature and some kinds of antibiotics. This suggests the hypothesis 408 that climate change might favor the evolution of resistance of specific (i.e. heat-similar) antibiotics indirectly by their resistance to high temperature stress. 
411 From our results it also appears that drugs can be used to modify temperature response curves in

412 predictable ways. A temperature-drug system could perhaps be used to examine scenarios for

413 biological responses to climate change via a variety of thermal responses in a laboratory setting.

414 Going forward, such a system could serve as a simplified model for examining changes in

415 response to temperature across seasons, geographic gradients, and climate change.

417 Temperature is one of the fundamental drivers of biological processes. By using antibiotics as

418 stressors, our study system is particularly valuable for its tractability, reproducibility, and

419 potential to study temperature-stressor interactions beyond the pairwise level. Our results provide

420 insights into the interactions between temperature and other stressors. Particularly, we show that

421 stressors can modify the temperature response curves of a living organism, and that these

422 changes can be predicted from the way the stressor harms its physiology. More broadly, our

423 results imply that the chemical environment - or potentially the presence of other stressors-for

424 a living organism can influence how it interacts with both abiotic (temperature) and biotic

425 (modified competition due to changes in its thermal niche) factors. Investigating stressor effects

426 on the physiological and ecological trait responses to temperature changes under this framework

427 could lead to future research directions in exploring other environmental stressors that may aid in

428 predicting the stability and diversity of ecological systems.

$431 \quad$ Materials and Methods

\section{Experimental Framework}

\section{$434 \quad$ Bacterial strain and growth medium}

435 The study used BW25113, a derivative of the F-, $\lambda-$, E. coli K-12 strain BD792 (CGSC6159)

436 (65). Bacterial cultures were grown in LB broth $(10 \mathrm{~g} / \mathrm{L}$ tryptone, $5 \mathrm{~g} / \mathrm{L}$ yeast extract, and $10 \mathrm{~g} / \mathrm{L}$ $437 \mathrm{NaCl}$ ) and maintained in $25 \%$ glycerol at $-80^{\circ} \mathrm{C}$. Fresh cultures were started by adding $20 \mu \mathrm{L}$ of 
thawed bacterial glycerol stock into $2 \mathrm{~mL}$ of LB followed by incubation at $37^{\circ} \mathrm{C}$. Cultures were grown to exponential growth phase and diluted to maintain $10^{4}$ cells per experimental condition.

Antibiotics

441 A total of 12 antibiotics were included in the study as representatives of all major drug classes.

442 Ciprofloxacin (CPR) from MP Biomedicals (Santa Ana, CA) and Gentamycin (GEN),

443 levofloxacin (LVX), tetracycline (TET), tobramycin (TOB), erythromycin (ERY), ampicillin

444 (AMP), clindamycin (CLI), streptomycin (STR), nitrofurantoin (NTR), cefoxitin (FOX), and

445 trimethoprim (TMP) —all from Sigma (St Louis, MO)—were used. Stock solution at $20 \mathrm{mg} / \mathrm{mL}$

446 of each antibiotic was stored in $50 \mu \mathrm{L}$ aliquots at $-20^{\circ} \mathrm{C}$. Each aliquot was only frozen and

447 thawed once to preserve potency.

\section{$448 \quad$ Growth experiments}

449 Antibiotics used in all experiments inhibited bacterial growth at sub-lethal concentrations (50\%

450 to $90 \%$ growth). The desired concentrations were first determined by a twelve-step concentration

451 series of 2-fold at each step in 96-well plates (Costar). Antibiotic stock solutions were prepared

452 in a total volume of $5 \mathrm{~mL}$ at 10 -fold of their respective concentrations (Table 1). Experiments of

453 pairwise drug combinations were prepared by adding $10 \mu \mathrm{L}$ of each component drug followed by

454 the addition of $80 \mu \mathrm{L}$ cell inoculum. $10 \mu \mathrm{L}$ of $\mathrm{LB}$ medium was added in replacement of a second

455 drug for single drug experiments. Each experimental condition was conducted in 4 replicates

456 from the same antibiotic stock solution. The plates were incubated at various temperatures

$457\left(22^{\circ} \mathrm{C}, 25^{\circ} \mathrm{C}, 30^{\circ} \mathrm{C}, 37^{\circ} \mathrm{C}, 41^{\circ} \mathrm{C}, 44^{\circ} \mathrm{C}, 46^{\circ} \mathrm{C}\right)$ with aeration at $300 \mathrm{rpm}$. Cell density was

458 measured at 4-hours, 8-hours, 12-hours and 24-hours by reading at OD600 $\mathrm{nm}$. The optical

459 density measurements (used as a proxy for bacterial growth) at 24-hours were used to infer the

460 temperature curves.

\section{Mathematical Framework}

\section{Extended Briere model for characterizing temperature response curves}

464 Briere (66) defines a simple model for the temperature dependence of a trait, such as growth, 465 denoted by $g(T)$ as follows: 


$$
g(T)=c\left(T-T_{\min }\right)\left(T-T_{\max }\right)^{\frac{1}{2}}
$$

466 where $T_{\min }, T_{\max }$ are the minimum and maximum temperature of growth. This equation can be 467 solved analytically (see Supplementary Material) to show that the optimal temperature yielding

468 maximum growth is always attained at $T_{\text {opt }}=\frac{2}{3} T_{\max }+\frac{1}{3} T_{\min }$. This model is not flexible

469 enough to describe the antibiotic growth curves we found empirically. As a more general

470 alternative, we propose an extended Briere model

$$
g(T)=c\left(T-T_{\min }\right)^{a}\left(T-T_{\max }\right)^{b}
$$

471 where $a, b \geq 0$ are parameters that determine the shape of the curve. In this extended Briere

472 model of temperature dependence of a trait, we have $T_{o p t}=\alpha T_{\max }+(1-\alpha) T_{\min }$. In this

473 model the optimum temperature can lie anywhere between $T_{\min }$ and $T_{\max }$ depending on the

474 value of the fraction $\alpha:=\frac{a}{a+b}$.

475 The extended Briere model can be reparametrized as

$$
g(T)=g_{\max }\left[\left(\frac{T-T_{\min }}{\alpha}\right)^{\alpha}\left(\frac{T_{\max }-T}{1-\alpha}\right)^{1-\alpha}\left(\frac{1}{T_{\max }-T_{\min }}\right)\right]^{S}
$$

476 where $g_{\max }$ is the maximum value of the trait, i.e. growth, and $s=a+b$ is a parameter that

477 determines the smoothness of the temperature response curve (see Supplementary Material for

478 details). We use this parametrization for parameter fitting of temperature response curves of the

479 bacterium across different drug combination treatments (see Table 1 for chosen drugs in our

480 study).

\section{Bayesian parameter fitting}

483 The extended Briere model was fitted to the temperature growth curve for the bacterium under

484 all conditions through a Bayesian methodology with the pymc3 library of the Python

485 programming language (67). The following methodology is used for obtaining Bayesian

486 estimates for the model parameters. Let $y_{i}$ be the $i$ th observed data point for growth after 24

487 hours and let $T_{i}$ be the temperature at which it was observed. The observed values were assumed

488 to be Gamma distributed with 
489

490

491

492

493

$$
y_{i} \mid g\left(T_{i}\right), \sigma_{T_{i}} \sim \operatorname{Gamma}\left(\mu=g\left(T_{i}\right), \quad \sigma=\sigma_{T_{i}}\right)
$$

where the Gamma distribution is parametrized in terms of the mean $\mu$ and standard deviation $\sigma$.

The data are clearly heteroskedastic, and multiple measurements were taken at the same

temperature enabling estimates of the standard deviation at each measured temperature. Because of this, a different standard deviation was modeled for each measured temperature. The following hierarchical model was used for the standard deviation:

$$
\begin{gathered}
\sigma_{T_{i}} \mid \beta \sim \text { halfCauchy }(\beta) \\
\beta \sim \operatorname{halfCauchy}(0.3)
\end{gathered}
$$

494 The prior distributions for the extended Briere model parameters and a justification for all priors 495 used is given in the Supplementary Material. A variational method (full-rank ADVI) (68) was 496 used to obtain approximate posterior distributions for the model parameters. These posterior 497 distributions were used to construct point estimates - the expected value of the posterior 498 distribution - and $95 \%$ credible intervals for all parameters to evaluate the uncertainty in the 499 estimates. Credible intervals are the Bayesian analog to confidence intervals. A credible interval 500 contains the true value of the parameter of interest with the specified (e.g. 95\%) probability, 501 given the observed data.

\section{Models for predicting optimal temperatures for multi-drug responses}

504 We denote single drugs as $X$ and $Y$, and the combination of drugs as $X Y$. We use these drug notations as a subscript for the corresponding optimal temperatures, i.e. $T_{o p t, X}, T_{o p t, Y}$, and

$506 T_{o p t, X Y}$. For predicting the optimal temperature of multi-drug combination treatments, we define 507 five different models by the choice of simple yet biologically meaningful scenarios (Figure 3).

508 (i) A single drug is playing a major role in determining the optimal temperature of the bacterial response. The optimal temperature of the combination is given by either of the two individual stressors ( $\min$ or max model). $T_{o p t, X Y}=\min \left(T_{o p t, X}, T_{o p t, Y}\right)$ or $T_{\text {opt }, X Y}=\max \left(T_{o p t, X}, T_{o p t, Y}\right)$.

(ii) The optimal temperature of the bacterium in the presence of drug combinations is shifted to lower or higher temperatures than both single drugs' optimal temperature 
values. Along the lines of these extreme behaviors, we define attenuated and elevated

(iii) Temperature tolerance is determined by both of the drugs in the combination. To uncover such cases, we define our fifth model, namely the mean optimal temperature model. This model expresses the optimal temperature of the combined treatment as

523 To determine the best optimal temperature model, we measured the difference between the actual

524 value and the predicted value of each of the min, mean, and max models. We considered the best-

525 fit model as the one with the smallest absolute difference between actual and predicted values.

526 When this absolute difference is greater than the cutoff value of $2.20^{\circ} \mathrm{C}$ (see Supplementary

527 Figure 2), then the best model is determined to be either the attenuated or elevated model

528 depending on the direction of the optimal temperature shift.

530 Data availability The dataset and code used in this manuscript will be made available upon

531 publication and for peer review upon request.

Acknowledgements We thank Nina Singh for comments on the manuscript. We thank Rina

534 Watanabe for laboratory assistance. We are grateful for funding from the Hellman Foundation

535 (PJY), a KL2 Fellowship (PJY) through the NIH/National Center for Advancing Translational

536 Science (NCATS) UCLA CTSI Grant Number UL1TR001881, UC Mexus and CONACYT

537 (MCL), and a James F. McDonnell Foundation Complex Systems Scholar Award (VMS).

\section{References:}

541 1. Chadwick LE, Rahn H. Temperature Dependence of Rattling Frequency in the 542 Rattlesnake, Crotalus v. viridis. Science. 1954;119(3092):442-3.

$5432 . \quad H u e y$ RB, Kingsolver JG. Evolution of thermal sensitivity of ectotherm performance.

544 Trends Ecol Evol. 1989;4(5):131-5. 
3. Lynch M, Gabriel W. Environmental tolerance. Am Nat. 1987;129(2):283-303.

4. Fenberg PB, Self A, Stewart JR, Wilson RJ, Broooks SJ. Exploring the universal ecological responses toclimate change in a univoltine butterfly. J Animal Ecol. 2016;85(3):739-48.

$548 \quad 5 . \quad$ Somero G. The physiology of climate change: how potentials for acclimatization and genetic adaptation will determine 'winners' and 'losers'. J Exp Biol. 2010;213(6):912-20. adaptations promote ecological divergence in a sympatric species pair of temperate freshwater fish, Coregonus spp. Funct Ecol. 2008;22:501-8.

553 7. Bennett AF. The thermal dependence of lizard behaviour. Anim Behav. 1980;28(3):752-

55462.

5558 8. Savage VM, Gilloly JF, Brown JH, Charmov EL. Effects of body size and temperature on

556 population growth. Am Nat. 2004;163(3):429-41.

557 9. Ali RM. The influence of suspension density and temperature on the filtration rate of 558 Hiatella arctica. Mar Biol. 1970;6(4):291-302.

559 10. Padfield D, Yvon-Durocher G, Buckling A, Jennings S, Yvon-Durocher G. Rapid evolution 560 of metabolic triats explains thermal adaptation in phytoplankton. Ecol Lett. 2016;19:133-42.

561 11. Parmesan C. Ecological and evolutionary responses to recent climate change. Annu Rev 562 Ecol Evol Syst. 2006;37:637-69.

563 12. Beaugrand G, Reid PC, Ibanez F, Lindley JA, Edwards M. Reorganization of North Atlantic marine copepod biodiversity and climate. Science. 2002;296(5573):1692-4. of their Thermal Tolerance. Integr Comp Biol. 2016;56(1):98-109.

14. Angilletta MJ. Thermal adaptation: a theoretical and empirical synthesis. Oxford Scholarship Press2009.

15. Angilletta MJ, Bennett AF, Guderley H, Navas CA, Seebacher F, Wilson RS. Coadaptation: a unifying principle in evolutionary thermal biology. Physiol Biochem Zool. 2006;79(2):282-94. and thermal tolerances in an invasive species across a climatic gradient: lessons from the land snail Cornu aspersum. PLoS One. 2013;8(8):e70662. physiological and ecological traits. Proc Natl Acad Sci USA. 2011;108(26):10591-6. 18. Russell NJ, Sandercock SP. The regulation of bacterial membrane fluidity by modification of phospholipid fatty acyl chain length. Membrane Fluidity 1: Humana Press; 1980.

580 20. Maeda K, Imae Y, Shioi JI, Oosawa F. Effect of temperature on motility and chemotaxis 581 of Escherichia coli. J Bacteriol. 1976;127(3):1039-46.

582 21. Paulick A, Jakovljevic V, Zhang S, Erickstad M, Groisman A, Meir Y, et al. Mechanism of 583 bidirectional thermotaxis in Escherichia coli. elife. 2017;6:e26607.

584 22. Takami H, Takaki Y, Chee GJ, Nishi S, Shimamura S, Suzuki H, et al. Thermoadaptation trait revealed by the genome sequence of thermophilic Geobacillus kaustophilus. Nucleic Acids 586 Res. 2004;32(21):6292-303.

587 23. Liao $\mathrm{H}$, Mackenzie $\mathrm{T}$, Hageman R. Isolation of a thermo- stable enzyme variant by cloning 588 and selection in a thermophile. Proc Natl Acad Sci USA. 1986;84:576-80. 
24. Chen Z, Farrell AP, Matala A, Narum SR. Mechanisms of thermal adaptation and evolutionary potential of conspecific populations to changing environments. Mol Ecol. 2018;27(3):659-74.

592 25. Richter K, Haslbeck M, Buchner J. The heat shock response: life on the verge of death. 593 Mol Cell. 2010;40(2):253-66.

594 26. Mayer MP, and Bukau, B. Hsp70 chaperones: cellular functions and molecular mechanism. Cell Mol Life Sci. 2005;62:670-84.

596 27. Reed TE, Schindler DE, Waples RS. Interacting effects of phenotypic plasticity and evolution on population persistence in a changing climate. Conserv Biol. 2011;25(1):56-63. 28. Thomas MK, Aranguren-Gassis M, Kremer CT, Gould MR, Anderson K, Klausmeier CA, et al. Temperature-nutrient interactions exacerbate sensitivity to warming in phytoplankton. Glob Change Biol. 2017;23:3269-80.

601 29. Martin TL, Huey RB. Why "suboptimal" is optimal: Jensen's inequality and ectotherm thermal preferences. Am Nat. 2008;171(3):E102-18.

603 30. Deutsch CA, Tewksbury JJ, Huey RB, Sheldon KS, Ghalambor CK, Haak DC, et al. Impacts of climate warming on terrestrial ectotherms across latitude. Proc Natl Acad Sci USA. 2008;105(18):6668-72.

606 31. Amarasekare P, Savage V. A framework for elucidating the temperature dependence of 607 fitness. Am Nat. 2012;179(2):178-91.

608 32. Cruz-Loya M, Kang TM, Lozano NA, Watanabe R, Tekin E, Damoiseaux R, et al. Stressor interaction networks suggest antibiotic resistance co-opted from stress responses to temperature. ISME J. 2019;13(1):12-23.

33. Vabulas RM, Raychaudhuri S, Hayer-Hartl M, Hartl FU. Protein folding in the cytoplasm and the heat shock response. Cold Spring Harb Perspect Biol. 2010;2(12):a004390. 34. VanBogelen RA, Neidhardt FC. Ribosomes as sensors of heat and cold shock in Escherichia coli. Proc Natl Acad Sci U S A. 1990;87(15):5589-93. promotes stress granule formation and cell survival in response to cold shock. Mol Biol Cell.

618 36. Sinha AK, Pavankumar TL, Kamisetty S, Mittal P, Ray MK. Replication arrest is a major 619 threat to growth at low temperature in Antarctic Pseudomonas syringae Lz4W. Mol Microbiol. $620 \quad 2013 ; 89(4): 792-810$.

$621 \quad 37 . \quad$ Lewis K. Persister cells, dormancy and infectious disease. Nat Rev Microbiol.

622 2007;5(1):48-56.

623 38. Mitchell SM, Ullman JL, Teel AL, Watts RJ. pH and temperature effects on the hydrolysis 624 of three beta-lactam antibiotics: ampicillin, cefalotin and cefoxitin. Sci Total Environ. 2014;466625 467:547-55.

626 39. Loughman K, Hall J, Knowlton S, Sindeldecker D, Gilson T, Schmitt DM, et al.

627 Temperature-Dependent Gentamicin Resistance of Francisella tularensis is Mediated by Uptake 628 Modulation. Front Microbiol. 2016;7:37.

629 40. Yeh P, Tschumi Al, Kishony R. Functional classification of drugs by properties of their 630 pairwise interactions. Nat Genet. 2006;38(4):489-94.

631 41. Segrè D, Deluna A, Church GM, Kishony R. Modular epistasis in yeast metabolism. Nat 632 Genet. 2005;37(1):77-83. 
633 42. Rodriguez-Verdugo A, Gaut BS, Tenaillon O. Evolution of Escherichia coli rifampicin 634 resistance in an antibiotic-free environment during thermal stress. BMC Evol Biol. 2013;13:50. 43. Greulich P, Scott M, Evans MR, Allen RJ. Growth-dependent bacterial susceptibility to ribosome-targeting antibiotics. Mol Syst Biol. 2015;11(3):796-.

637

44. Mingeot-Leclercq MP, Glupczynski Y, Tulkens PM. Aminoglycosides: activity and resistance. Antimicrob Agents Chemother. 1999;43(4):727-37. 45. VanBogelen RA, Neidhardt FC. Ribosomes as Sensors of Heat and Cold Shock in Escherichia coli. Proc Natl Acad Sci USA. 1990;87(15):5589-93.

46. Okie JG, Van Horn DJ, Storch D, Barrett JE, Gooseff MN, Kopsova L, et al. Niche and metabolic principles explain patterns of diversity and distribution: theory and a case study with soil bacterial communities. Proc Biol Sci. 2015;282(1809):20142630.

644 47. Fischer K, Dierks A, Franke K, Geister TL, Liszka M, Winter S, et al. Environmental effects on temperature stress resistance in the tropical butterfly Bicyclus anynana. PLoS One. 2010;5(12):e15284.

647 48. Brennan G, Collins S. Growth responses of a green alga to multiple environmental 648 drivers. Nat Clim Change. 2015;5(9):892-7.

649 49. Rosso L, Lobry JR, Bajard S, Flandrois JP. Convenient Model To Describe the Combined 650 Effects of Temperature and pH on Microbial Growth. Appl Environ Microbiol. 1995;61(2):610-6. 651 50. Kovárová K, Zehnder AJ, Egli T. Temperature-dependent growth kinetics of Escherichia 652 coli ML 30 in glucose-limited continuous culture. J Bacteriol. 1996;178(15):4530-9.

653 51. Parra R, Magan N. Modelling the effect of temperature and water activity on growth of 654 Aspergillus niger strains and applications for food spoilage moulds. J Appl Microbiol.

655 2004;97(2):429-38.

656 52. Cuppers HG, Oomes S, Brul S. A model for the combined effects of temperature and salt concentration on growth rate of food spoilage molds. Appl Environ Microbiol.

658 1997;63(10):3764-9.

659 53. Mlot C. Microbiology. Antibiotics in nature: beyond biological warfare. Science.

660 2009;324(5935):1637-9.

661 54. Martínez JL. Antibiotics and antibiotic resistance genes in natural environments.

662 Science. 2008;321(5887):365-7.

663 55. Dietrich LE, Teal TK, Price-Whelan A, Newman DK. Redox-active antibiotics control gene 664 expression and community behavior in divergent bacteria. Science. 2008;321(5893):1203-6.

665 56. Huey RB, Kingsolver JG. Variation in universal temperature dependence of biological 666 rates. Proc Natl Acad Sci USA. 2011;108(26):10377-8.

667 57. Rohr JR, Civitello DJ, Cohen JM, Roznik EA, Sinervo B, Dell Al. The complex drivers of 668 thermal acclimation and breadth in ectotherms. Ecol Lett. 2018;21(9):1425-39.

669 58. MacFadden DR, McGough SF, Fisman D, Santillana M, Brownstein JS. Antibiotic 670 resistance increases with local temperature. Nat Clim Change. 2018;8(6):510-4.

671 59. Gautam R, Bani-Yaghoub M, Neill WH, Dopfer D, Kaspar C, Ivanek R. Modeling the effect 672 of seasonal variation in ambient temperature on the transmission dynamics of a pathogen with 673 a free-living stage: example of Escherichia coli 0157:H7 in a dairy herd. Prev Vet Med. 674 2011;102(1):10-21.

675 60. Mermel LA, Machan JT, Parenteau S. Seasonality of MRSA infections. PLoS One. 676 2011;6(3):e17925. 
677 61. Lorenz MG, Wackernagel W. Bacterial gene transfer by natural genetic transformation in 678 the environment. Microbiol Rev. 1994;58(3):563-602.

679 62. Walsh TR, Weeks J, Livermore DM, Toleman MA. Dissemination of NDM-1 positive

680 bacteria in the New Delhi environment and its implications for human health: an environmental 681 point prevalence study. Lancet Infect Dis. 2011;11(5):355-62.

682 63. Molnar PK, Kutz SJ, Hoar BM, Dobson AP. Metabolic approaches to understanding

683 climate change impacts on seasonal host-macroparasite dynamics. Ecol Lett. 2013;16(1):9-21.

684 64. Harvell CD, Mitchell CE, Ward JR, Altizer S, Dobson AP, Ostfeld RS, et al. Climate

685 Warming and Disease Risks for Terrestrial and Marine Biota. Science. 2002;296(5576):2158-62.

686 65. Datsenko KA, Wanner BL. One-step inactivation of chromosomal genes in

687 \&lt;em\&gt; Escherichia coli\&lt;/em\&gt; K-12 using PCR products. Proc Natl Acad Sci USA.

688 2000;97(12):6640.

689 66. Briere J-F, Pracos P, Roux A-YL, Pierre J-S. A Novel Rate Model of Temperature-

690 Dependent Development for Arthropods. Environ Entomol. 1990;28(1):22-9.

691 67. Salvatier J, Wiecki TV, Fonnesbeck C. Probabilistic programming in Python using PyMC3.

692 PeerJ Preprints. 2016.

693 68. Kucukelbir A, Tran D, Ranganath R, Gelman A, Blei DM. Automatic differentiation

694 variational inference. J Mach Learn Res. 2017;18(1):430-74.

695 
696 Table 1. List of antibiotics. The antibiotics used are listed with their abbreviation, mechanism

697 of action, dose, and our color scheme throughout the paper. Similar colors are chosen for drugs

698 belonging to the same class/mechanism of action. For example, colors of blue tones are chosen

699 for aminoglycosides. The similarity of each antibiotic to temperature stress according to their

700 interactions with other stressors (32) is also shown, along with the corresponding range of

701 growth temperatures that show similarity. For the purposes of cold/heat similarity, we consider

702 any antibiotic with a similar temperature range lower than the optimum as cold-similar. For

703 example, there are two groups of cold-similar antibiotics, which we call cold $\left(22-37^{\circ} \mathrm{C}\right)$ and very

704 cold $\left(22-25^{\circ} \mathrm{C}\right)$. These terms are used to distinguish the groups by the relative strength of the cold

705 stress they are similar to, but not necessarily the severity of the cold stress in an absolute sense.

\begin{tabular}{|c|c|c|c|c|c|}
\hline Antibiotic & Abbreviation & Color & Mechanism of Action & $\begin{array}{l}\text { Dose } \\
(\mu g / m L)\end{array}$ & $\begin{array}{l}\text { Temperature } \\
\text { similarity }\end{array}$ \\
\hline Ampicillin & AMP & & $\begin{array}{l}\text { cell wall synthesis } \\
\text { inhibitor }\end{array}$ & 1.2 & None \\
\hline Cefoxitin & FOX & & $\begin{array}{l}\text { cell wall synthesis } \\
\text { inhibitor }\end{array}$ & 1.2 & None \\
\hline Levofloxacin & LVX & & $\begin{array}{l}\text { fluoroquinolone, DNA } \\
\text { gyrase inhibitor }\end{array}$ & 0.01 & $\begin{array}{l}\text { cold } \\
\left(22-37^{\circ} \mathrm{C}\right)\end{array}$ \\
\hline Ciprofloxacin & CPR & & $\begin{array}{l}\text { fluoroquinolone, DNA } \\
\text { gyrase inhibitor }\end{array}$ & 0.005 & $\begin{array}{l}\text { cold } \\
\left(22-37^{\circ} \mathrm{C}\right)\end{array}$ \\
\hline Nitrofurantoin & NTR & & $\begin{array}{l}\text { DNA damaging, multiple } \\
\text { mechanisms }\end{array}$ & 2 & $\begin{array}{l}\text { hot } \\
\left(44^{\circ} \mathrm{C}\right)\end{array}$ \\
\hline Trimethoprim & TMP & & $\begin{array}{l}\text { folic acid synthesis } \\
\text { inhibitor }\end{array}$ & 0.1 & $\begin{array}{l}\text { hot } \\
\left(44^{\circ} \mathrm{C}\right)\end{array}$ \\
\hline Tobramycin & TOB & & aminoglycoside & 1.5 & $\begin{array}{l}\text { very hot } \\
\left(46^{\circ} \mathrm{C}\right)\end{array}$ \\
\hline Gentamycin & GEN & & aminoglycoside & 1 & $\begin{array}{l}\text { very hot } \\
\left(46^{\circ} \mathrm{C}\right)\end{array}$ \\
\hline Streptomycin & STR & & aminoglycoside & 2 & $\begin{array}{l}\text { very hot } \\
\left(46^{\circ} \mathrm{C}\right)\end{array}$ \\
\hline Clindamycin & CLI & & $\begin{array}{l}\text { protein synthesis } \\
\text { inhibitor, } 50 \mathrm{~S}\end{array}$ & 40 & $\begin{array}{l}\text { very cold } \\
\left(22-25^{\circ} \mathrm{C}\right)\end{array}$ \\
\hline Erythromycin & ERY & & protein synthesis & 50 & very cold \\
\hline
\end{tabular}


bioRxiv preprint doi: https://doi.org/10.1101/2020.04.04.025874; this version posted April 6, 2020. The copyright holder for this preprint (which was not certified by peer review) is the author/funder, who has granted bioRxiv a license to display the preprint in perpetuity. It is made available under aCC-BY-NC-ND 4.0 International license.

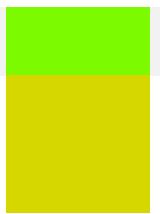

inhibitor, $50 \mathrm{~S}$

protein synthesis

inhibitor, 30S $\left(22-25^{\circ} \mathrm{C}\right)$

0.25 cold

$\left(22-37^{\circ} \mathrm{C}\right)$

707

708 
Figure 1. Temperature response curves change under antibiotic stress. (a) An example of a

710 left shift of optimal temperature with antibiotic GEN. (b) An example of a right shift of optimal

711 temperature with antibiotic ERY. (c) Optimal growth temperature (middle dot) and temperature

712 niche (thin line joining the half-maximal growth temperatures, left and right dots) observed

713 under each antibiotic used in this study. Point estimates for the optimal and half-maximal growth

714 temperatures are shown as dots. To show the uncertainty in the estimates, $95 \%$ credible intervals

715 (CIs, see Materials and Methods) are drawn as thick lines. The CIs for the no drug condition are

716 shaded in the plot to facilitate comparison.

(a)

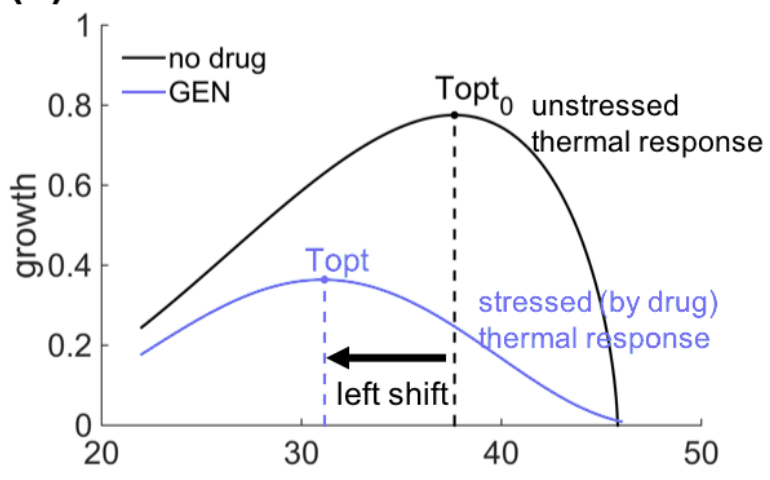

(b)

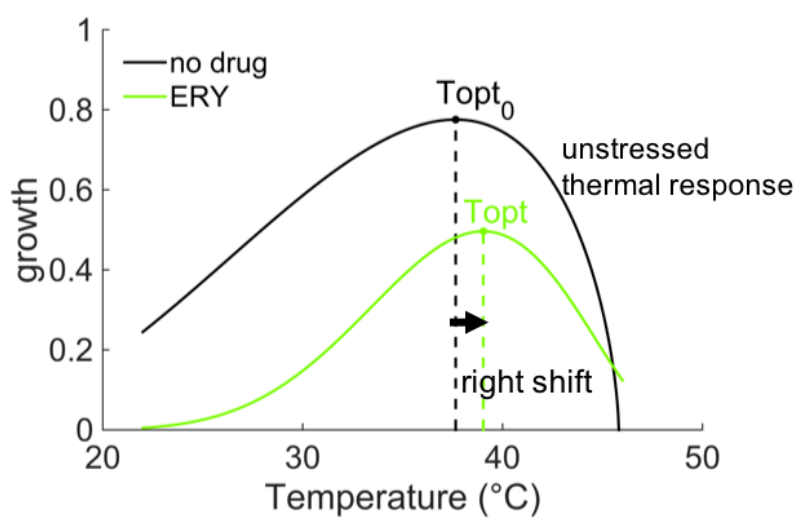

(c)

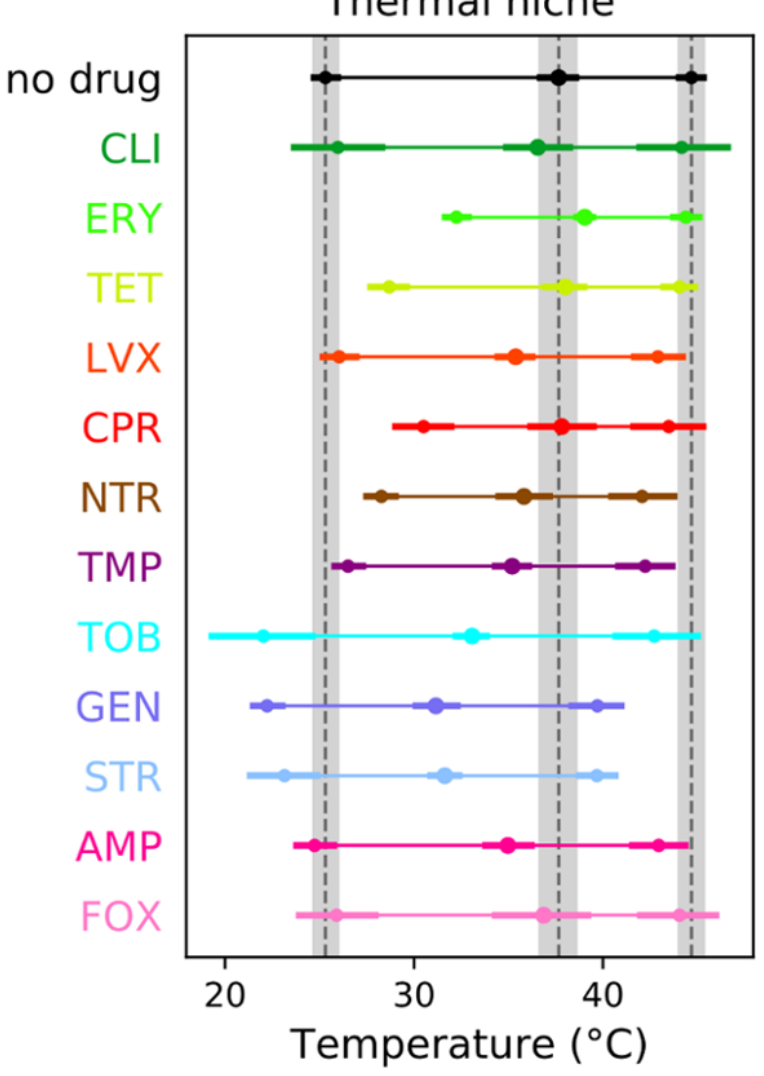


Figure 2. Physiological effects of antibiotics predict the direction of shifts in the optimal

temperature. (a) Left: The fitted temperature response curve in the presence of single antibiotics is compared to the unstressed growth condition. Drugs are grouped according to the

722 similarity of their effects to temperature (32), as shown in the top of the plots, except beta-

723 lactams which did not show similarity to temperature. Right: Histogram of shifts in the optimal

724 temperature under all pairwise drug combinations involving the drugs in the group. The

725 individual estimates are shown as lines in the bottom. The unstressed optimal temperature is

726 shown as a dotted line in both sets of plots. For both single drugs and combinations, the direction

727 of the optimal temperature shifts depends on whether the drug is similar to cold or heat. (b)

728 Optimal growth temperature and temperature niche observed under each antibiotic combination

729 used in this study. The first drug in the combination is shown at the top of the plot. The second

730 drug is shown in the y-axis using its assigned line color. The CIs for the single drug conditions

731 are shown with shaded $95 \%$ credible intervals to facilitate comparisons. Conditions where the

732 maximum growth was too small to estimate parameters reliably were removed.

(a)
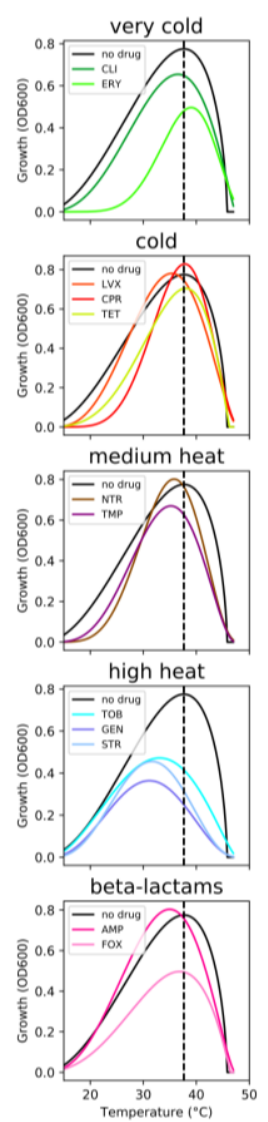

733 (b)
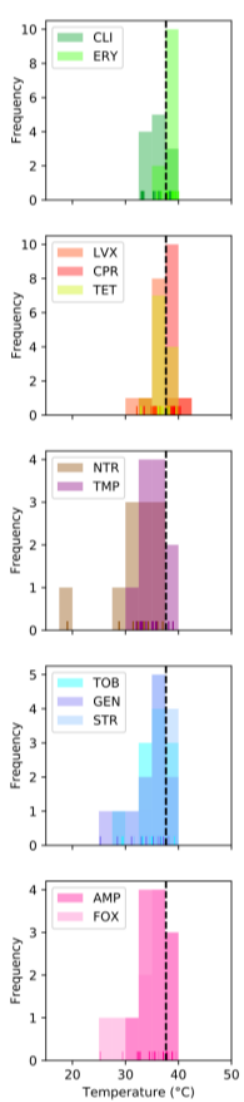
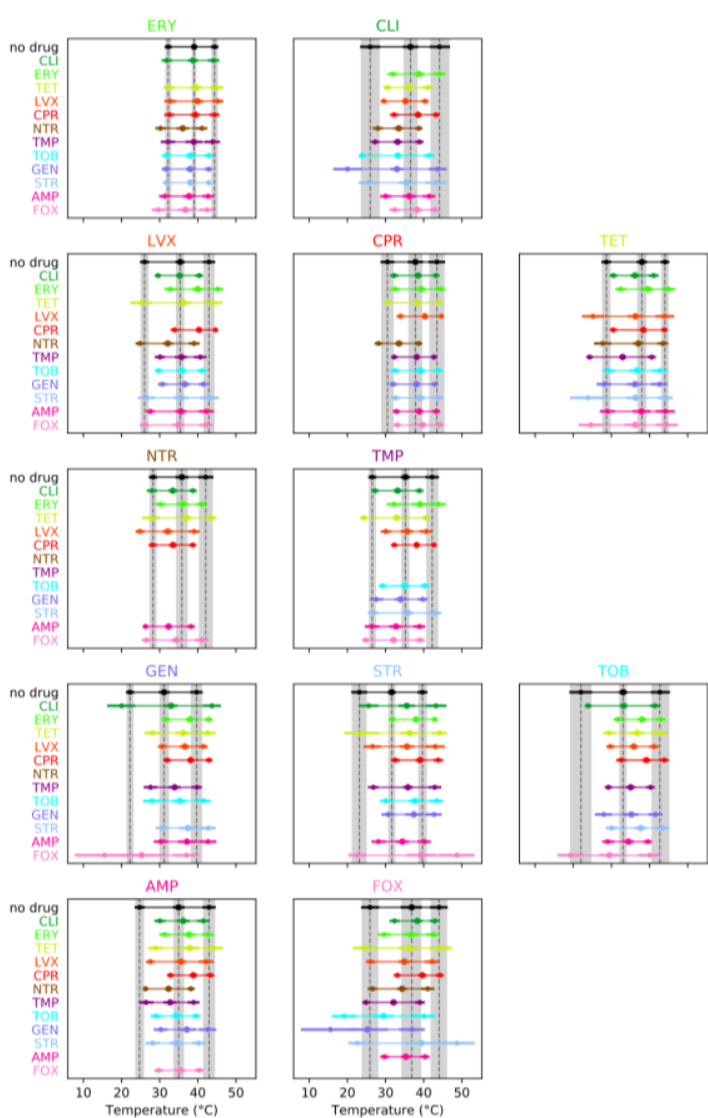


\section{Figure 3. The optimal growth temperature under stressor combinations is often determined}

735 by a single stressor. (a) Schematic illustration of models to determine the optimal growth

736 temperature under two stressors $\left(T_{o p t}, X Y\right)$ given the single stressor optimal temperatures

737 ( $\left.T_{\text {opt }, X}, T_{\text {opt }, Y}\right)$. (b) The frequency at which each model is the best fit, across all drug combinations. (c) Proportion of time each antibiotic is the main driver of the optimal temperature

739 when combined with other antibiotics, based on the individual models: min, max, attenuated, and 740 elevated.

(a)
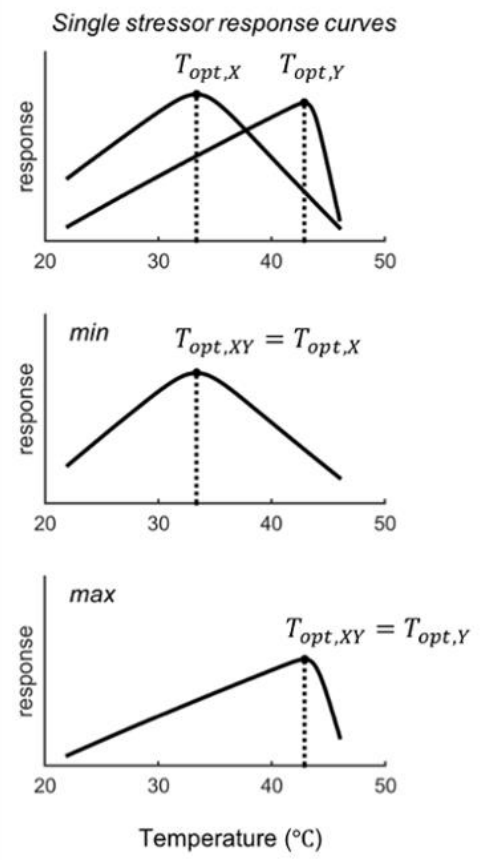
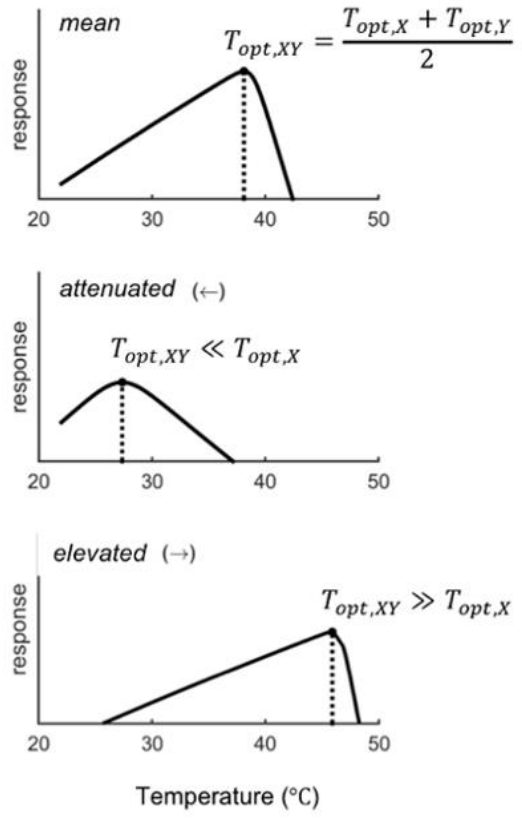

(b)

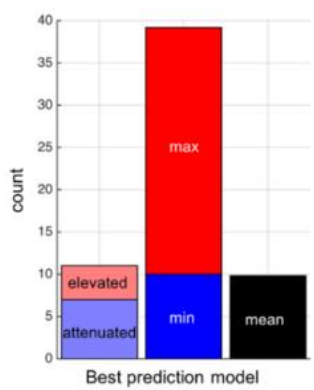

(c)

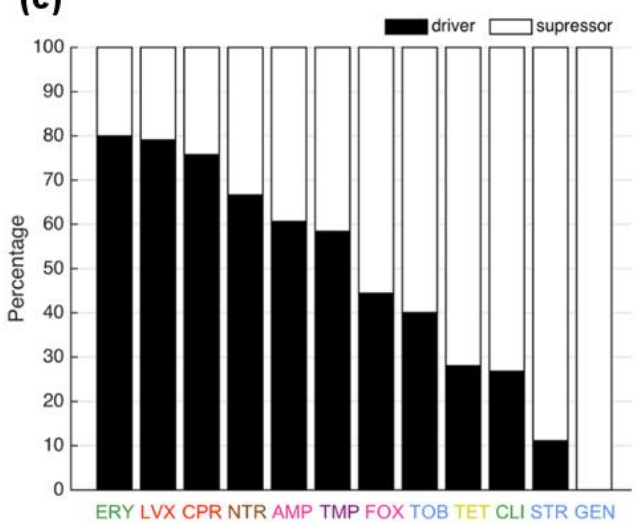


Supplemental Figure 1. Full dataset and model fits. Lower half: The growth data for all antibiotic combinations (blue dots), as well as the fitted extended Briere model (black lines), are shown, as well as $95 \%$ credible intervals for the extended Briere model. The upper left corner corresponds to the no drug case. Upper half: The fitted curves corresponding to each drug combination are shown in black, and the fits corresponding to each single drug are shown in their corresponding color. In the uppermost row, the growth curve in the absence of antibiotics is shown in black and the single drug curves are shown in the corresponding color.
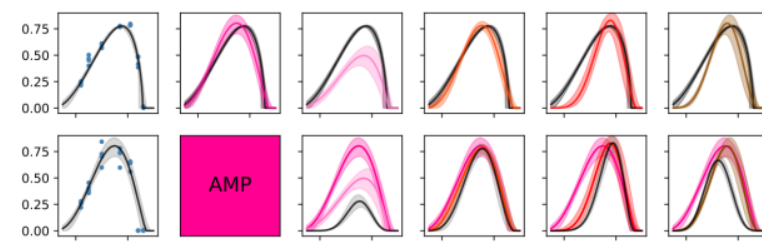

2.25 -
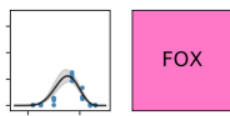

0.75
0.25
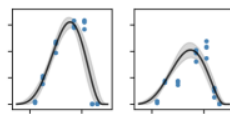

A $0.75-25$
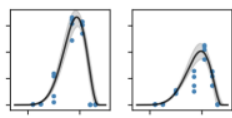

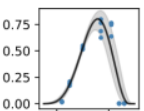

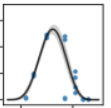

C.

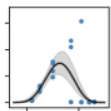

$0.25-7$

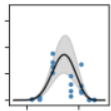

(

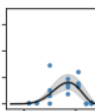

0.75

0.25 .7

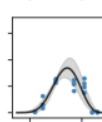

0.75

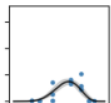

0.75
$0.50-25$
0.00

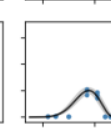

O. $0.50-25$
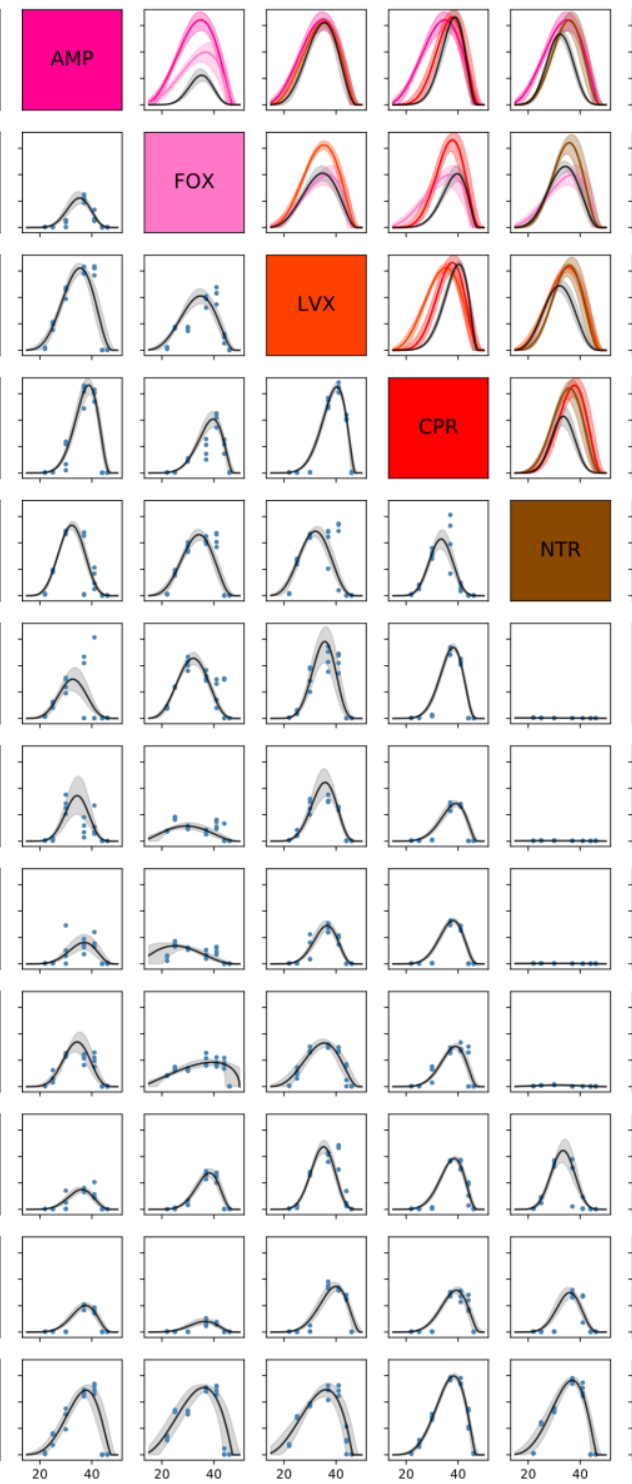
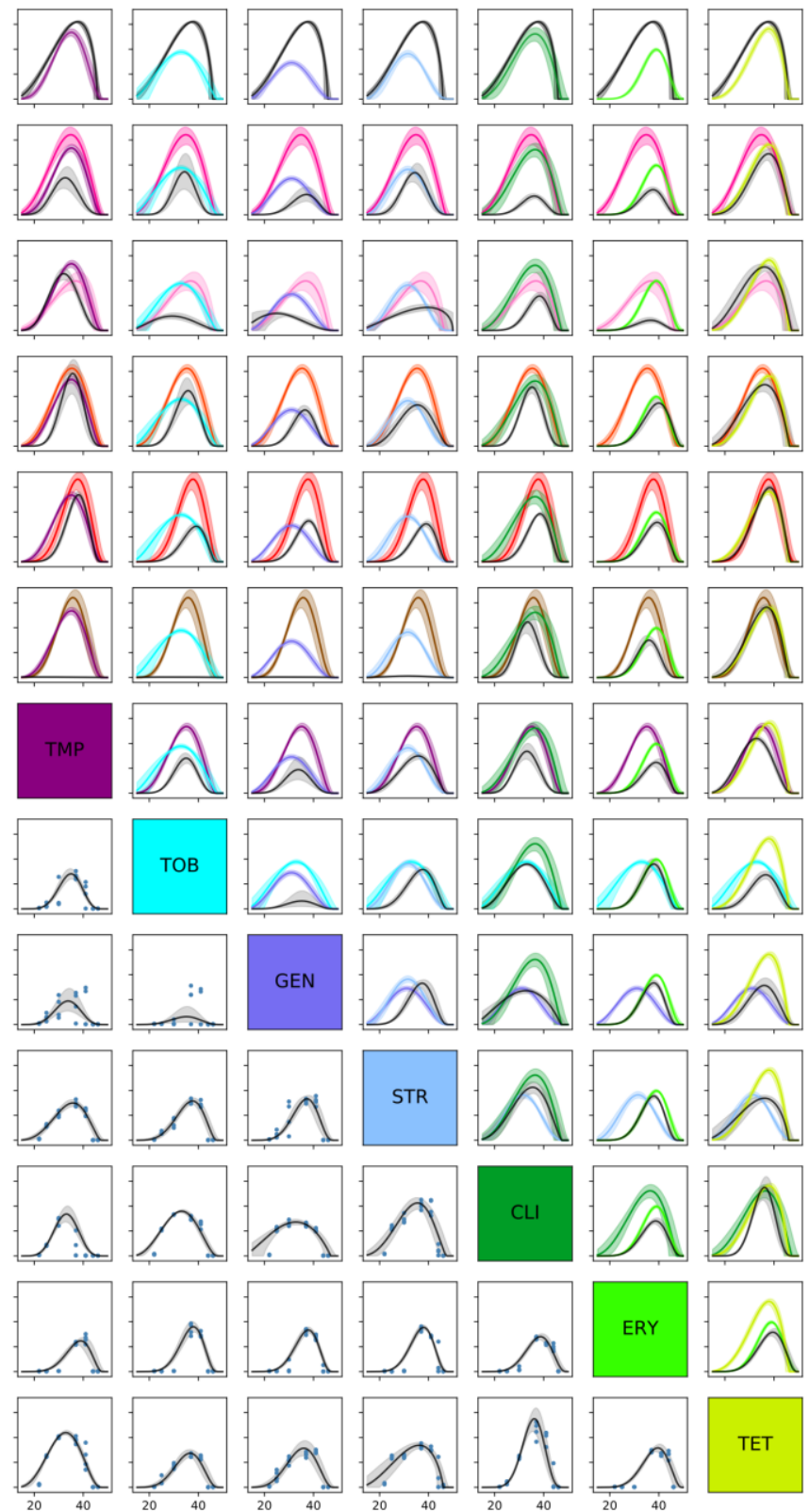

A
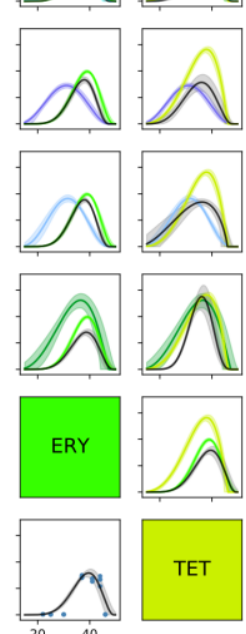


\section{Supplementary Figure 2. Threshold for distinguishing single-driver models (min versus}

755 attenuated or max versus elevated). The distribution of model prediction errors across the $\min$,

756 mean, and max models is plotted to decide a cutoff to determine if the optimal temperature shift

757 of a pairwise combination is large enough to decide the attenuated or elevated models are a better

758 fit. When the prediction error (as defined by an absolute value of difference of optimal

759 temperature prediction and actual optimal temperature) is higher than $2.20{ }^{\circ} \mathrm{C}$, the best model is

760 either the attenuated model or elevated model based on the direction.

761

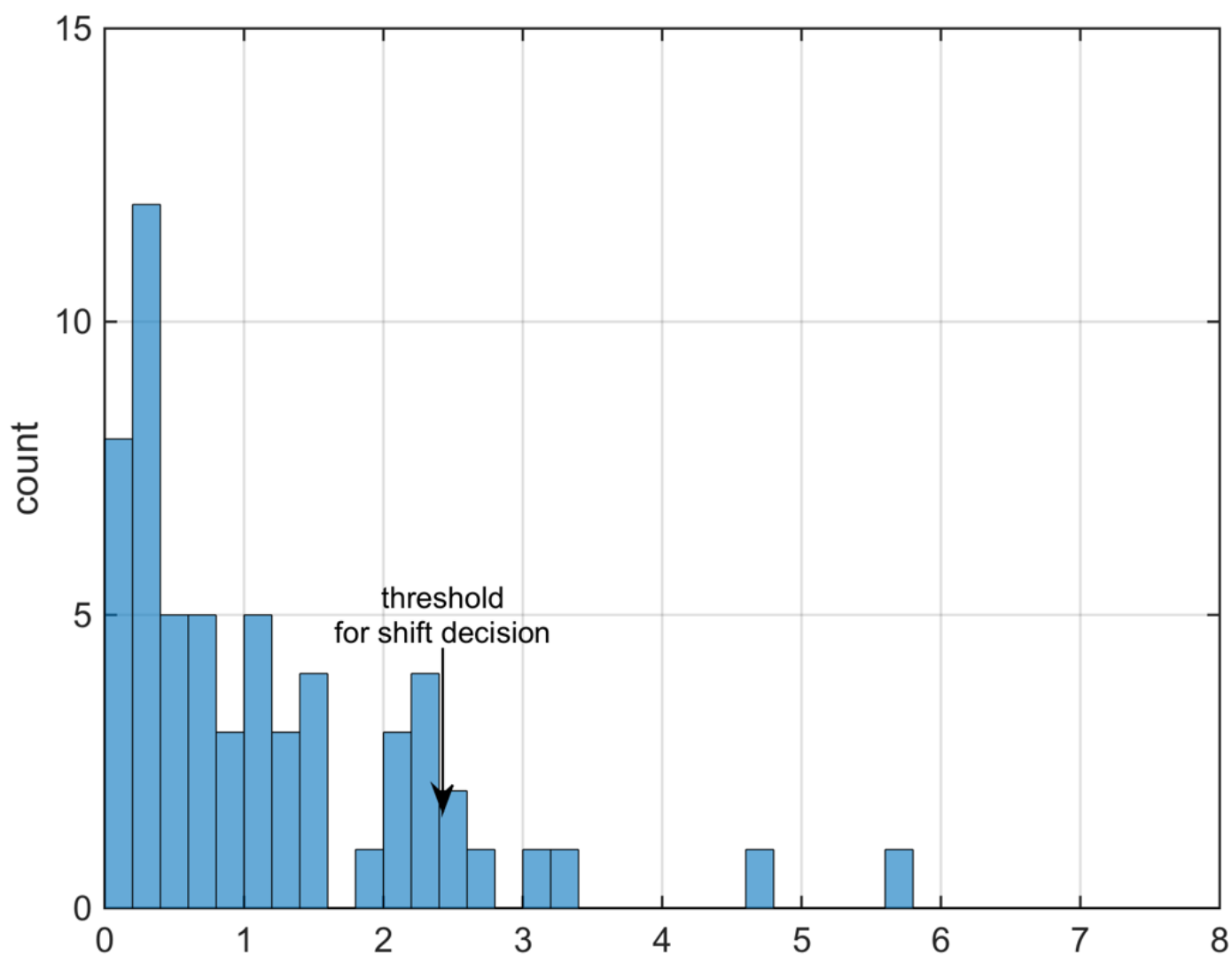


763 Supplementary Figure 3. The optimal temperature model yielding the best prediction for each

764 drug pair is shown. The blue oval frame represents the min model, the red rectangular frame

765 represents the max model, the light red circle frame with the arrow pointing right represents the

766 elevated model, and the light blue frame with the arrow pointing left represents the attenuated

767 model. Four pairs - specifically AMP+TOB, CLI+AMP, TMP+LVX, and CPR+TET-exhibited

768 multiple models showing the best model prediction, with all results showing mean and max to be

769 the best models. These pairs are displayed as "multiple" in the figure.

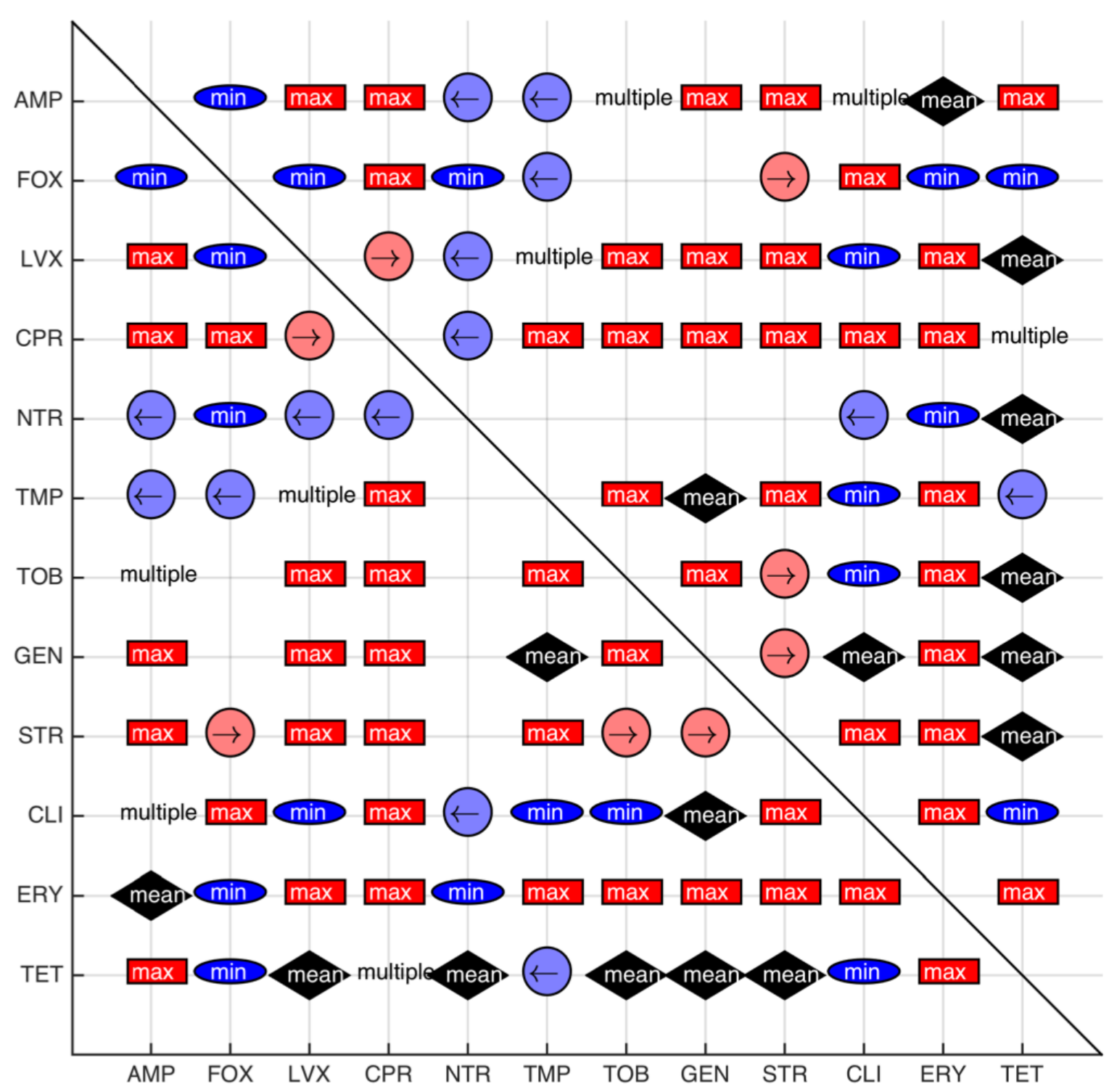

